\title{
Panobinostat synergizes with bortezomib to induce endoplasmic reticulum stress and ubiquitinated protein accumulation in renal cancer cells
}

\author{
Akinori Sato*, Takako Asano, Makoto Isono, Keiichi Ito and Tomohiko Asano
}

\begin{abstract}
Background: Inducing endoplasmic reticulum (ER) stress is a novel strategy used to treat malignancies. Inhibition of histone deacetylase (HDAC) 6 by the HDAC inhibitor panobinostat hinders the refolding of unfolded proteins by increasing the acetylation of heat shock protein 90 . We investigated whether combining panobinostat with the proteasome inhibitor bortezomib would kill cancer cells effectively by inhibiting the degradation of these unfolded proteins, thereby causing ubiquitinated proteins to accumulate and induce ER stress.

Methods: Caki-1, ACHN, and 769-P cells were treated with panobinostat and/or bortezomib. Cell viability, clonogenicity, and induction of apoptosis were evaluated. The in vivo efficacy of the combination was evaluated using a murine subcutaneous xenograft model. The combination-induced ER stress and ubiquitinated protein accumulation were assessed.

Results: The combination of panobinostat and bortezomib induced apoptosis and inhibited renal cancer growth synergistically (combination indexes $<1$ ). It also suppressed colony formation significantly $(p<0.05)$. In a murine subcutaneous tumor model, a 10-day treatment was well tolerated and inhibited tumor growth significantly ( $p$ <.05). Enhanced acetylation of the HDAC6 substrate alpha-tubulin was consistent with the suppression of HDAC6 activity by panobinostat, and the combination was shown to induce ER stress and ubiquitinated protein accumulation synergistically.
\end{abstract}

Conclusions: Panobinostat inhibits renal cancer growth by synergizing with bortezomib to induce ER stress and ubiquitinated protein accumulation. The current study provides a basis for testing the combination in patients with advanced renal cancer.

Keywords: Panobinostat, Bortezomib, Endoplasmic reticulum stress, Ubiquitinated protein, Histone acetylation, Renal cancer, Combination therapy

\section{Background}

A new therapeutic approach to advanced renal cancer is urgently needed because there is presently no curative treatment, and one innovative treatment strategy used against cancer is to induce endoplasmic reticulum (ER) stress and ubiquitinated protein accumulation [1]. Protein unfolding rates that exceed the capacity of protein chaperones cause ER stress, and chronic or unresolved ER stress

\footnotetext{
* Correspondence: zenpaku@ndmc.ac.jp

Department of Urology, National Defense Medical College, 3-2 Namiki, Tokorozawa, Saitama 359-8513, Japan
}

can lead to apoptosis [2]. On the other hand, unfolded proteins that fail to be repaired by chaperones are then ubiquitinated and the accumulation of these ubiquitinated proteins is also cytotoxic [3].

Histone deacetylase (HDAC) 6 inhibition acetylates heat shock protein (HSP) 90 and suppresses its function as a molecular chaperon, increasing the amount of unfolded proteins in the cell [4]. Because these unfolded proteins are then ubiquitinated and degraded by the proteasome [5], HDAC6 inhibition alone is thought to cause no or only slight ER stress and ubiquitinated protein 
accumulation if the proteasome function is normal. We thought that combining an HDAC inhibitor with the proteasome inhibitor bortezomib would cause ER stress and ubiquitinated protein accumulation synergistically because the increased ubiquitinated proteins would not be degraded by the inhibited proteasome.

Panobinostat is a novel HDAC inhibitor that has been clinically tested not only in patients with hematological malignancies [6,7] but also patients with solid tumors, including renal cell carcinoma [8,9]. Bortezomib has been approved by the FDA and widely used for the treatment of multiple myeloma [10].

In the present study using renal cancer cells, we investigated whether the combination of panobinostat and bortezomib induces ER stress and ubiquitinated protein accumulation, and kills cancer cells effectively in vitro and in vivo.

\section{Methods}

\section{Cell lines}

Renal cancer cell lines (Caki-1, ACHN, and 769-P) were purchased from the American Type Culture Collection (Rockville, MD). Caki-1 cells were maintained in MEM, ACHN cells in DMEM, and 769-P cells in RPMI medium, all supplemented with $10 \%$ fetal bovine serum and $0.3 \%$ penicillin-streptomycin (Invitrogen, Carlsbad, CA).

\section{Reagents}

Panobinostat and bortezomib were obtained from Cayman Chemical (Ann Arbor, MI) and LC Laboratories (Woburn, MA), respectively, dissolved in dimethyl sulfoxide (DMSO), and stored at $-20^{\circ} \mathrm{C}$ until use.

Evaluating effect of the combination of panobinostat and bortezomib on cell viability and colony formation

For cell viability assay, $5 \times 10^{3}$ cells were plated in a 96-well culture plate one day before treatment and treated with panobinostat (25-50 nM) and/or bortezomib (5-15 nM) for 48 hours. Cell viability was evaluated by MTS assay (Promega, Madison, WI) according to the manufacturer's protocol. For colony formation assay, $1 \times$ $10^{2}$ cells were plated in 6-well plates one day before treatment and cultured for 48 hours in media containing $50 \mathrm{nM}$ panobinostat and/or $10 \mathrm{nM}$ bortezomib. They were then given fresh media and allowed to grow for 1-2 weeks, depending on the cell line. The number of colonies was then counted after fixing the cells with $100 \%$ methanol and staining them with Giemsa's solution.

Evaluating effect of the combination of panobinostat and bortezomib on induction of apoptosis

$1.5 \times 10^{5}$ cells were plated in a 6 -well culture plate one day before being cultured for 48 hours in medium containing $50 \mathrm{nM}$ panobinostat and/or $10 \mathrm{nM}$ bortezomib.
Induction of apoptosis was evaluated, using flow cytometry, by annexin-V assay and cell cycle analysis. For annexin- $\mathrm{V}$ assay the harvested cells were stained with annexin $\mathrm{V}$ according to the manufacturer's protocol (Beckman Coulter, Marseille, France). For cell cycle analysis the harvested cells were resuspended in citrate buffer and stained with propidium iodide. They were then analyzed by flow cytometry using CellQuest Pro Software (BD Biosciences, San Jose, CA).

\section{Murine xenograft model}

The animal protocol for this experiment has been approved by the institutional Animal Care and Use Committee of National Defense Medical College. 5-week-old male nude mice (strain BALB/c Slc-nu/nu) were purchased from CLEA (Tokyo, Japan). The animals were housed under pathogen-free conditions and had access to standard food and water ad libitum. $1 \times 10^{7}$ Caki- 1 cells were subcutaneously injected into the flank and treatments were initiated 4 days after the injection (day 1 ), when the tumors became palpable. The mice were divided into 4 groups of 5, the control group receiving intraperitoneal injections of DMSO and the other groups receiving either panobinostat $(2 \mathrm{mg} / \mathrm{kg})$ or bortezomib $(60 \mu \mathrm{g} / \mathrm{kg})$ or both. Injections were given once a day, 5 days a week, for 2 weeks. Tumor volume was estimated as one half of the product of the length and the square of the width (i.e., volume $=0.5 \times$ length $\times$ width $^{2}$ ).

\section{Western blotting}

Cells were treated under the indicated conditions for 48 hours and whole-cell lysates were obtained using RIPA buffer. Equal amounts of protein were subjected to SDS-PAGE and transferred onto nitrocellulose membranes that were then probed with antibodies specific for glucose-regulated protein (GRP) 78, ubiquitin (Santa Cruz Biotechnology, Santa Cruz, CA), actin (Millipore, Billerica, MA), HSP70, endoplasmic reticulum resident protein (ERp) 44, endoplasmic oxidoreductin-1-like protein (Ero1-L) $\alpha$, cleaved poly(ADP-ribose) polymerase (PARP) (Cell Signaling Technology, Danvers, MA), acetylated $\alpha$-tubulin (Enzo Life Sciences, Farmingdale, NY), and acetylated histone (Abcam, Cambridge, UK). This probing was followed by treatment with horseradish-peroxidase-tagged secondary antibodies (Bio-Rad, Hercules, CA) and visualization by chemiluminescence (ECL, Amersham, Piscataway, NJ).

\section{Statistical analyses}

The combination indexes were calculated using the Chou-Talalay method and CalcuSyn software (Biosoft, Cambridge, UK). The statistical significance of observed differences between samples was determined using the Mann-Whitney U test (StatView software, SAS Institute, 
Cary, NC). Differences were considered significant at $\mathrm{p}<0.05$.

\section{Results}

Combination of panobinostat and bortezomib inhibited renal cancer growth synergistically

We first investigated the combined effect of panobinostat and bortezomib on renal cancer cell viability by MTS assay. Panobinostat and bortezomib each inhibited the growth of renal cancer cells in a dose-dependent fashion, and the combination did so more effectively than either did by itself (Figure 1A). Analysis using the Chou-Talalay method indicated that the effect of the combination was synergistic (combination index $<1$ ) in many of the treatment conditions (Table 1). We then investigated whether the combination affects the clonogenic survival of renal cancer cells. Colony formation assay revealed that the combination suppressed colony formation significantly and did so significantly more than did either panobinostat or bortezomib alone (Figure 1B).

We also used a subcutaneous xenograft mouse model to test the efficacy of the combination therapy in vivo. A 10-day treatment with panobinostat and bortezomib was well tolerated and suppressed tumor growth significantly (Figure 2). The $\mathrm{p}$ values at day 12 were 0.0283 for the control group and combination group, 0.0283 for the bortezomib group and combination group, and 0.0472 for the panobinostat group and combination group. The average tumor size at day 15 was $520 \pm 175 \mathrm{~mm}^{3}$ (mean \pm SE) in the vehicle-treated mice and was $266 \pm$ $39 \mathrm{~mm}^{3}$ in the combination-treated mice. Thus the combination of panobinostat and bortezomib was shown to be effective for suppressing renal cancer growth both in vitro and in vivo.
A
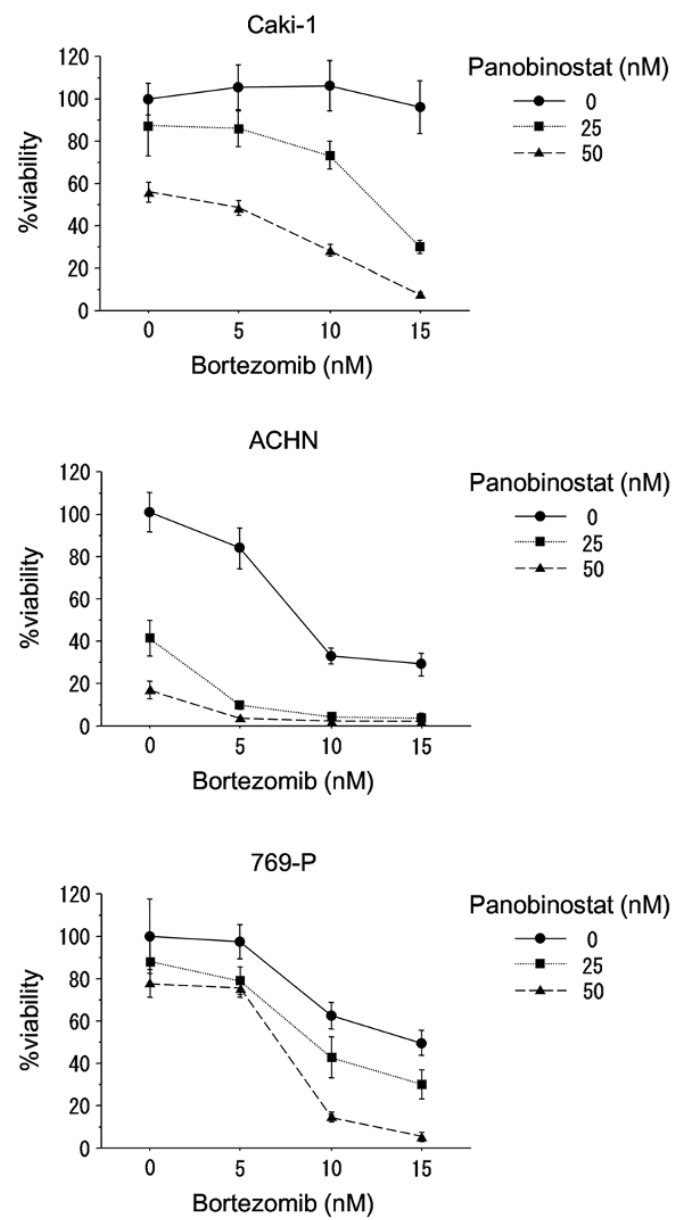

B
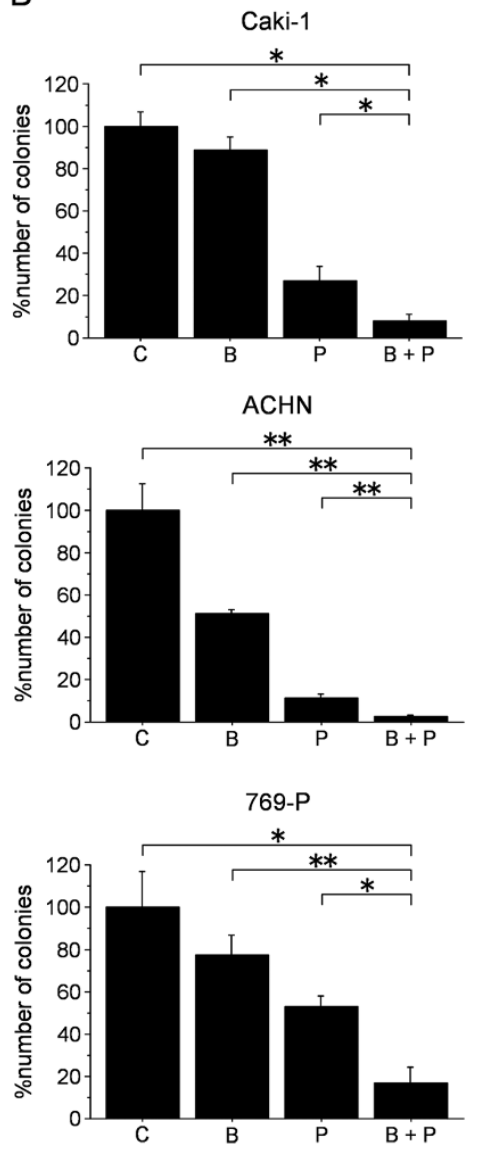

Figure 1 The combination of panobinostat and bortezomib inhibited renal cancer growth effectively. A, MTS assay results (mean \pm SD, $n=6$ ) after cells were treated for 48 hours either with bortezomib or panobinostat alone or with bortezomib and panobinostat together. B, Colony formation assay results (mean $\pm S D, n=3$ ) after $1-2$ week incubation in control media (C) or media containing $50 \mathrm{nM}$ panobinostat $(\mathrm{P})$ and/or $10 \mathrm{nM}$ bortezomib (B). ${ }^{*} p=0.0495 ;{ }^{* *} p=0.0463$. 
Table 1 Combination indexes for the combination of panobinostat and bortezomib in renal cancer cells

\begin{tabular}{cccc}
\hline Panobinostat (nM) & \multicolumn{3}{c}{ Bortezomib (nM) } \\
\cline { 2 - 4 } & $\mathbf{5}$ & $\mathbf{1 0}$ & $\mathbf{1 5}$ \\
\hline Caki-1 & & & \\
$\mathbf{2 5}$ & 0.927 & 0.581 & 0.791 \\
$\mathbf{5 0}$ & 1.186 & 0.737 & 0.808 \\
$\mathbf{A C H N}$ & & & \\
$\mathbf{2 5}$ & 0.557 & 0.458 & 0.553 \\
$\mathbf{5 0}$ & 0.463 & 0.394 & 0.544 \\
$\mathbf{7 6 9 - P}$ & & & \\
$\mathbf{2 5}$ & 1.074 & 0.803 & 0.946 \\
$\mathbf{5 0}$ & 1.41 & 0.512 & 0.519 \\
\hline
\end{tabular}

\section{Combination of panobinostat and bortezomib induced apoptosis}

The combination increased the annexin- $\mathrm{V}$ fluorescence intensity (up to 19.4-fold compared with control vehicle) (Figure 3A) and also increased the number of the cells in the sub-G1 fraction (up to 70.5\%) (Figure 3B). Thus the combination of panobinostat and bortezomib was demonstrated to induce apoptosis in renal cancer cells.

Combination of panobinostat and bortezomib induced ER stress and ubiquitinated protein accumulation synergistically

The combination induced ER stress synergistically as indicated by the increased expression of ER stress markers such as GRP78, HSP70, ERp44, and (except in 769-P cells) Ero1-L $\alpha$ (Figure 4A). As expected, the

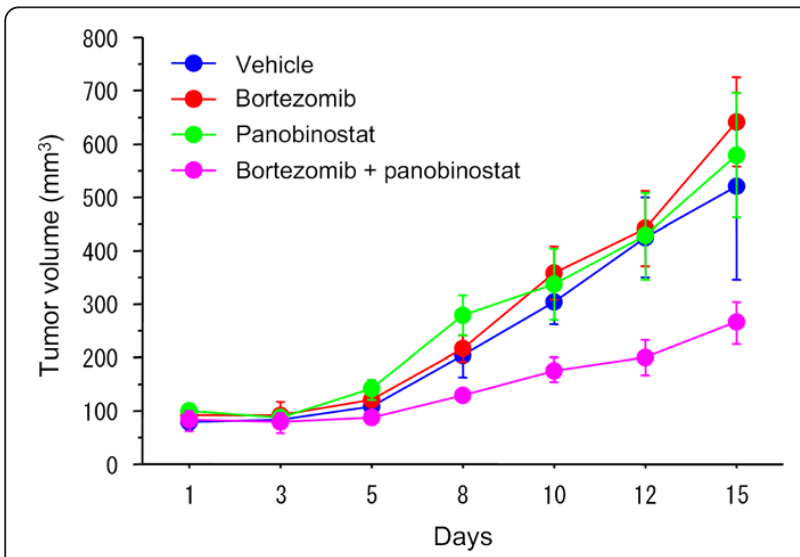

Figure 2 The combination of panobinostat and bortezomib suppressed tumor growth in vivo. A murine subcutaneous tumor model was made using Caki-1 cells, and the control group received intraperitoneal injections of DMSO, while other groups received either panobinostat $(2 \mathrm{mg} / \mathrm{kg})$ or bortezomib $(60 \mu \mathrm{g} / \mathrm{kg})$ or both. Injections were given once a day, 5 days a week, for 2 weeks. The 10-day treatment was well tolerated and suppressed tumor growth significantly (mean $\pm \mathrm{SE} ; \mathrm{p}=0.0283$ at day 12 ). combination induced ubiquitinated protein accumulation synergistically (Figure 4B): in Caki-1 and 769-P cells, $10 \mathrm{nM}$ bortezomib alone did not cause ubiquitinated proteins to accumulate but in combination with $50 \mathrm{nM}$ panobinostat increased the accumulation of ubiquitinated proteins markedly. In ACHN cells, $10 \mathrm{nM}$ bortezomib caused ubiquitinated protein accumulation and the accumulation was synergistically enhanced by $50 \mathrm{nM}$ panobinostat. Acetylation of $\alpha$-tubulin by panobinostat is consistent with HDAC6 inhibition because $\alpha$-tubulin is one of the important substrates of HDAC6. Interestingly, the combination also enhanced the acetylation of histone and $\alpha$-tubulin synergistically in Caki- 1 and ACHN cells. In 769-P cells, the combination enhanced the acetylation of $\alpha$-tubulin but not that of histone.

\section{Histone acetylation was a consequence of ubiquitinated protein accumulation}

We then investigated the relationship between histone acetylation and ubiquitinated protein accumulation. Panobinostat caused histone acetylation in a dose-dependent fashion in all the cell lines but did not induce ubiquitinated protein accumulation (Figure 5A). Bortezomib, on the other hand, caused both ubiquitinated protein accumulation and histone acetylation in a dose-dependent fashion in Caki-1 and ACHN cells but did not cause histone acetylation in 769-P cells (Figure 5B). This is in accordance with the result that the combination did not enhance histone acetylation in 769-P cells despite inducing ubiquitinated protein accumulation in them (Figure 4B). We inferred from these results that the histone acetylation the combination caused in Caki-1 and ACHN cells was a consequence of ubiquitinated protein accumulation.

\section{Discussion}

Inducing ER stress and ubiquitinated protein accumulation is a novel approach to cancer therapy. The combination of an HDAC inhibitor and bortezomib is one of the combinations that might be expected to do it. The combination of panobinostat and bortezomib has recently been investigated mainly in hematological malignancies $[11,12]$. It has been reported that the combination of bortezomib and the HDAC inhibitor suberoylanilide hydroxamic acid inhibits renal cancer growth by causing accumulation of ubiquitinated proteins and histone acetylation [13], but that study did not show the relationship between ubiquitinated protein accumulation and histone acetylation. In the present study, using panobinostat, a more potent HDAC inhibitor (acting at nanomolar concentrations, whereas suberoylanilide hydroxamic acid acts at micromolar concentrations), we investigated the effect of the bortezomib-panobinostat combination on renal cancer growth as well as further mechanisms of the combination of bortezomib and an HDAC inhibitor. 
A
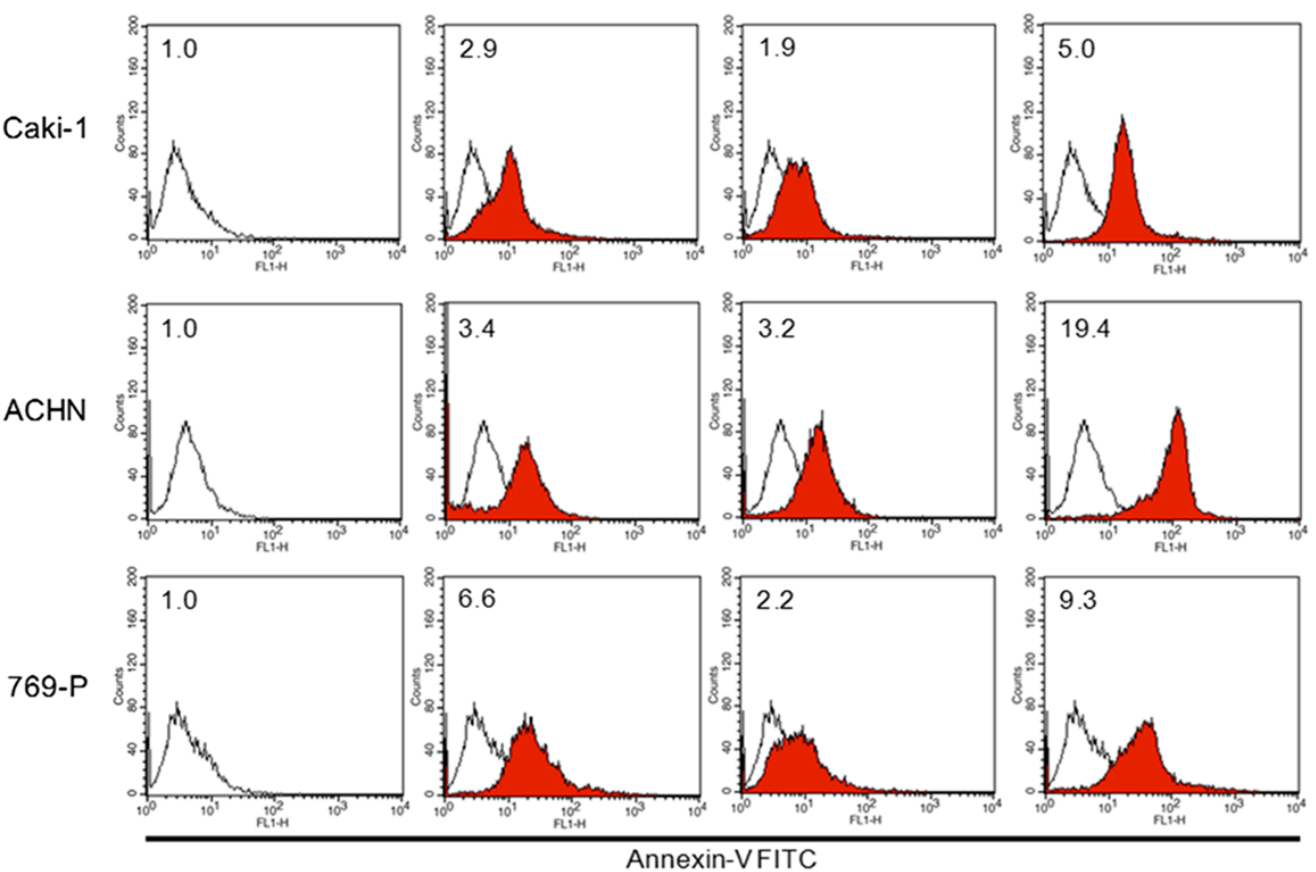

$10 \mathrm{nM}$ bortezomib

$50 \mathrm{nM}$ panobinostat
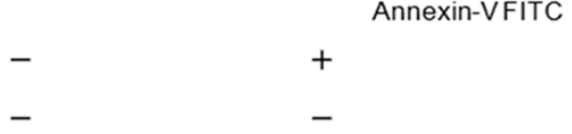

B

$10 \mathrm{nM}$ bortezomib
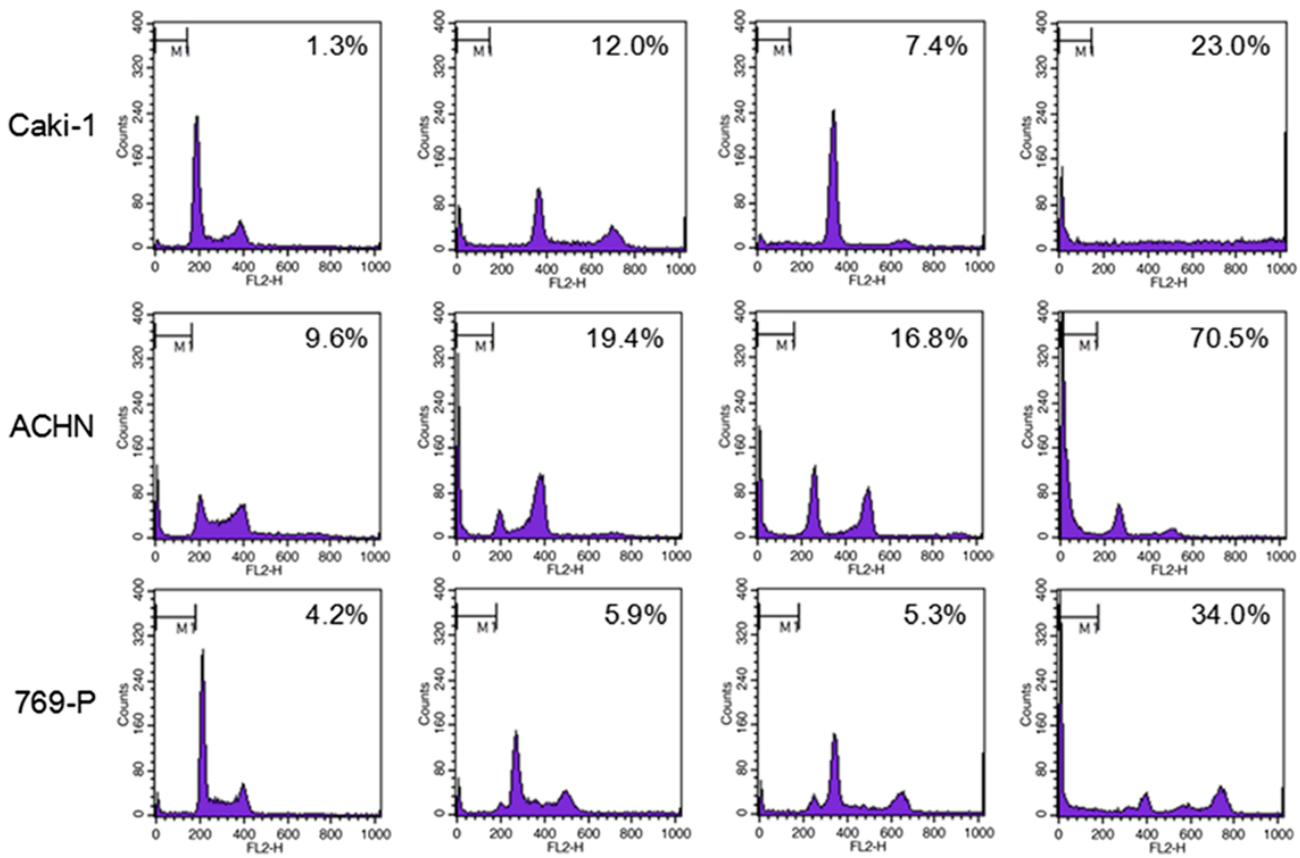

$50 \mathrm{nM}$ panobinostat

Figure 3 (See legend on next page.) 
(See figure on previous page.)

Figure 3 The combination of panobinostat and bortezomib induced apoptosis in renal cancer cells. Cells were treated for 48 hours with $50 \mathrm{nM}$ panobinostat with or without $10 \mathrm{nM}$ bortezomib. The combination increased the annexin-V-FITC fluorescence intensity (A) and increased the number of the cells in the sub-G1 fraction (B). Relative annexin-V-FITC fluorescence intensity (control = 1) is shown in the insets. White, control; red, treated. The percentage of cells in the sub-G1 fraction is shown in the graph. Representative results are shown.

Inhibition of HDAC6 acetylates HSP90, abrogating its function and increasing the amount of unfolded proteins [4]. We think that bortezomib inhibits degradation of unfolded proteins increased by panobinostat, which induces ER stress and ubiquitinated protein accumulation. Accumulation of unfolded proteins, or ER stress, activates a signaling pathway known as the unfolded protein response (UPR), which leads to increased transcription of ER folding and quality-control factors [14]. In the present study we showed the induction of ER stress by detecting the increased expression of UPR-related proteins: GRP78, HSP70, Ero1-L $\alpha$, and ERp44. GRP78 is a master regulator for ER stress because of its role as a major ER chaperone as well as its ability to control the activation of UPR signaling [15]. HSP70 is a molecular chaperone localized in the cytoplasm but associated with the regulation of the UPR by forming a stable protein complex with the cytosolic domain of inositol-requiring enzyme $1 \alpha$ [16]. Ero1-L $\alpha$ regulates oxidative protein folding by selectively oxidizing protein disulfide isomerase [17], one of the key players in the control of disulfide bond formation [18]. ERp44 forms mixed disulfides with Ero1-L $\alpha$ and may be involved in the control of oxidative protein folding [19]. The increased expression of these ER stress-related proteins thus confirmed that ER stress was induced by the combination of panobinostat and bortezomib.

Acetylation of $\alpha$-tubulin, one of the important substrates of HDAC6 [20], is consistent with the inhibition of HDAC6 by panobinostat. Interestingly, panobinostat itself did not cause marked ER stress even though it inhibited HDAC6 function. This may be because the






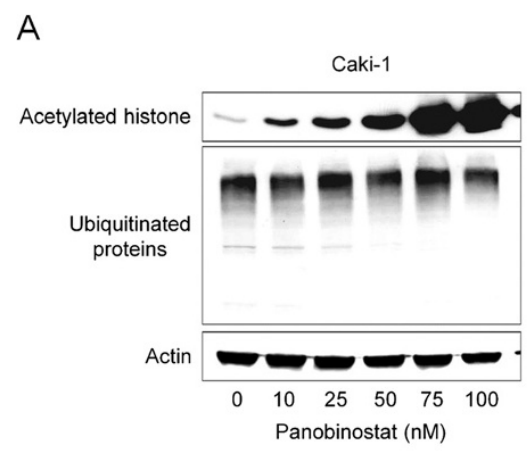

B

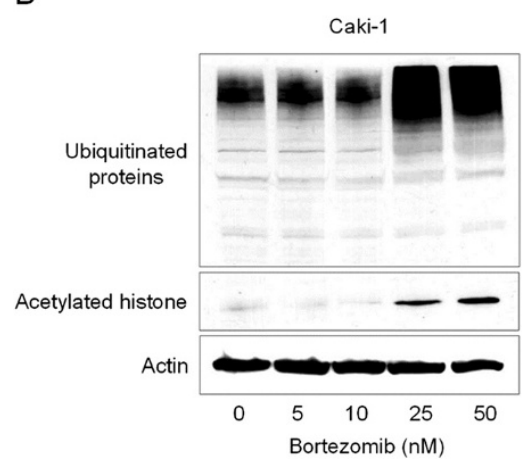




Figure 5 Histone acetylation was a consequence of ubiquitinated protein accumulation. A, 48-hour treatment with panobinostat caused dose-dependent histone acetylation in all the cell lines but did not cause ubiquitinated protein accumulation. B, 48-hour treatment with bortezomib, on the other hand, caused both histone acetylation and ubiquitinated protein accumulation in Caki-1 and ACHN cells and caused only ubiquitinated protein accumulation in 769-P cells.

unfolded proteins increased by panobinostat can be degraded immediately by the proteasome if its function is not suppressed. This explanation is consistent with the result that panobinostat induced marked ER stress only when combined with bortezomib.

The combination induced ubiquitinated protein accumulation synergistically. This is because panobinostat increased unfolded proteins, which were then ubiquitinated, and bortezomib inhibited their degradation. The ubiquitinated protein accumulation is also in accordance with the above-discussed enhanced ER stress induced by the combination because ER stress is induced by the accumulation of unfolded proteins in the cell, and many of these unfolded proteins are ubiquitinated. Not only are ubiquitinated proteins themselves toxic to tumor cells [3], some of them may be important molecules for cancer cell survival (such as transcription factors and signal transduction molecules) that have lost their function because of unfolding and ubiquitination, presumably leading to the inhibition of multiple signal transduction pathways. Furthermore, the inhibition of NF-kB is thought to play an important role in the combination therapy with panobinostat and bortezomib because of the accumulation of undegraded IkB, a suppressor of NF-kB [21]. Jiang XJ et al. reported that the combination of panobinostat and bortezomib activated caspases and down-regulated antiapoptotic proteins such as XIAP and Bcl-2 through inhibition of the AKT and NF-kB pathways [11]. The combination is thus thought to inhibit cancer growth by diverse mechanisms other than the induction of ER stress and ubiquitinated protein accumulation.

In Caki-1 and ACHN cells the combination of panobinostat and bortezomib not only caused ubiquitinated protein accumulation but also enhanced histone acetylation. In these cell lines, panobinostat alone caused histone acetylation but not ubiquitinated protein accumulation, whereas bortezomib alone induced both ubiquitinated protein accumulation and histone acetylation. We therefore think the histone acetylation in these cell lines is a consequence of ubiquitinated protein accumulation, which is consistent with the results of a previous study using prostate cancer cells [22]. In 769-P cells, on the other hand, the combination enhanced ubiquitinated protein accumulation but not histone acetylation. This is, however, also in accordance with the result that bortezomib alone did not cause histone acetylation in 769-P cells. In Caki-1 and ACHN cells, HDAC function decreased by ubiquitination may be one explanation. In 769-P cells, bortezomib alone seems to even decrease histone acetylation. Ubiquitination may result in the HDAC 
activity in 769-P cells being higher than the histone acetyltransferase activity there. However, further study will be needed to clarify the exact mechanism of this decreased histone acetylation.

The combination of panobinostat and bortezomib has also been tested clinically, mainly in patients with hematological malignancies. In the most recent phase-II study enrolling 55 patients with relapsed and bortezomibrefractory myeloma [23], the patients were treated with eight 3-week cycles of $20 \mathrm{mg}$ panobinostat three times a week and $1.3 \mathrm{mg} / \mathrm{m}^{2}$ bortezomib twice a week with $20 \mathrm{mg}$ of dexamethasone four times a week on weeks 1 and 2. If the patients showed clinical benefit, then they were treated with 6-week cycles of panobinostat three times a week and bortezomib once a week on weeks 1,2 , and 4 with dexamethasone on the days of and after bortezomib. In that study the overall response rate was $34.5 \%$, the clinical benefit rate was $52.7 \%$, and grade 3 or 4 adverse events were thrombocytopenia (63.6\%), fatigue (20.0\%), and diarrhea (20.0\%). Two limitations of our in-vivo study are that it could not provide information about whether the doses we used in mice were equivalent to those used in humans and that it lacked a proper assessment of side effects. This study is, however, the first to show the beneficial combined effect of panobinostat and bortezomib in renal cancer cells, and it provides a basis for testing the combination in clinical settings.

\section{Conclusions}

Panobinostat inhibits renal cancer growth by synergizing with bortezomib to induce ER stress and ubiquitinated protein accumulation. Histone acetylation may be another important mechanism of action. This is the first study to demonstrate the combination's effect on renal cancer cells both in vitro and in vivo, and it provides a basis for testing the combination in patients with advanced renal cancer.

\section{Competing interests}

The authors declare that they have no competing interests.

\section{Authors' contributions}

AS contributed to design and interpretation of all experiments, drafting of the manuscript and execution of western blotting, colony formation assay and animal experiments. TA collected and assembled data and performed MTS assay, cell cycle analysis, annexin- $V$ assay and animal experiments. Ml participated in the study design, performed statistical analysis and helped to draft the manuscript. KI contributed to design and interpretation of all experiments and drafting of the manuscript. TA participated in the study design and coordination and helped to draft the manuscript. All authors read and approved the final manuscript.

Received: 21 March 2014 Accepted: 26 August 2014

Published: 30 August 2014

\section{References}

1. Liu Y, Ye Y: Proteostasis regulation at the endoplasmic reticulum: a new perturbation site for targeted cancer therapy. Cell Res 2011, 21:867-883.

2. Tabas I, Ron D: Integrating the mechanisms of apoptosis induced by endoplasmic reticulum stress. Nat Cell Biol 2011, 13:184-190.
3. Mimnaugh EG, Xu W, Vos M, Yuan X, Isaacs JS, Bisht KS, Gius D, Neckers L: Simultaneous inhibition of hsp 90 and the proteasome promotes protein ubiquitination, causes endoplasmic reticulum-derived cytosolic vacuolization, and enhances antitumor activity. Mol Cancer Ther 2004, 3:551-566.

4. Bali P, Pranpat M, Bradner J, Balasis M, Fiskus W, Guo F, Rocha K Kumaraswamy S, Boyapalle S, Atadja P, Seto E, Bhalla K: Inhibition of histone deacetylase 6 acetylates and disrupts the chaperone function of heat shock protein 90: a novel basis for antileukemia activity of histone deacetylase inhibitors. J Biol Chem 2005, 280:26729-26734.

5. Glickman MH, Ciechanover A: The ubiquitin-proteasome proteolytic pathway: destruction for the sake of construction. Physiol Rev 2002, 82:373-428.

6. Duvic M, Dummer R, Becker JC, Poulalhon N, Ortiz Romero P, Grazia Bernengo M, Lebbé C, Assaf C, Squier M, Williams D, Marshood M, Tai F, Prince HM: Panobinostat activity in both bexarotene-exposed and -naïve patients with refractory cutaneous T-cell lymphoma: results of a phase II trial. Eur J Cancer 2013, 49:386-394.

7. Wolf JL, Siegel D, Goldschmidt H, Hazell K, Bourquelot PM, Bengoudifa BR, Matous J, Vij R, de Magalhaes-Silverman M, Abonour R, Anderson KC, Lonial S: Phase II trial of the pan-deacetylase inhibitor panobinostat as a single agent in advanced relapsed/refractory multiple myeloma. Leuk Lymphoma 2012, 53:1820-1823.

8. Morita S, Oizumi S, Minami H, Kitagawa K, Komatsu Y, Fujiwara Y, Inada M, Yuki S, Kiyota N, Mitsuma A, Sawaki M, Tanii H, Kimura J, Ando Y: Phase I dose-escalating study of panobinostat (LBH589) administered intravenously to Japanese patients with advanced solid tumors. Invest New Drugs 2012, 30:1950-1957.

9. Hainsworth JD, Infante JR, Spigel DR, Arrowsmith ER, Boccia RV, Burris HA: A phase II trial of panobinostat, a histone deacetylase inhibitor, in the treatment of patients with refractory metastatic renal cell carcinoma. Cancer Invest 2011, 29:451-455.

10. Kane RC, Farrell AT, Sridhara R, Pazdur R: United States Food and Drug Administration approval summary: bortezomib for the treatment of progressive multiple myeloma after one prior therapy. Clin Cancer Res 2006, 12:2955-2960.

11. Jiang XJ, Huang KK, Yang M, Qiao L, Wang Q, Ye JY, Zhou HS, Yi ZS, Wu FQ, Wang ZX, Zhao QX, Meng FY: Synergistic effect of panobinostat and bortezomib on chemoresistant acute myelogenous leukemia cells via AKT and NF-KB pathways. Cancer Lett 2012, 326:135-142.

12. Rao R, Nalluri S, Fiskus W, Savoie A, Buckley KM, Ha K, Balusu R, Joshi A, Coothankandaswamy V, Tao J, Sotomayor E, Atadja P, Bhalla KN: Role of CAAT/ enhancer binding protein homologous protein in panobinostat-mediated potentiation of bortezomib-induced lethal endoplasmic reticulum stress in mantle cell lymphoma cells. Clin Cancer Res 2010, 16:4742-4754.

13. Sato A, Asano T, Ito $K$, Sumitomo M, Asano T: Suberoylanilide hydroxamic acid (SAHA) combined with bortezomib inhibits renal cancer growth by enhancing histone acetylation and protein ubiquitination synergistically. BJU Int 2012, 109:1258-1268.

14. Mori K: Tripartite management of unfolded proteins in the endoplasmic reticulum. Cell 2000, 101:451-454.

15. Lee AS: The ER chaperone and signaling regulator GRP78/BiP as a monitor of endoplasmic reticulum stress. Methods 2005, 35:373-381.

16. Gupta S, Deepti A, Deegan S, Lisbona F, Hetz C, Samali A: HSP72 protects cells from ER stress-induced apoptosis via enhancement of IRE1alpha-XBP1 signaling through a physical interaction. PLOS Biol 2010, 8:e1000410.

17. Mezghrani A, Fassio A, Benham A, Simmen T, Braakman I, Sitia R: Manipulation of oxidative protein folding and PDI redox state in mammalian cells. EMBO J 2001, 20:6288-6296.

18. Bulleid NJ, Freedman RB: Defective co-translational formation of disulphide bonds in protein disulphide-isomerase-deficient microsomes. Nature 1988, 335:649-651.

19. Anelli T, Alessio M, Mezghrani A, Simmen T, Talamo F, Bachi A, Sitia R: ERp44, a novel endoplasmic reticulum folding assistant of the thioredoxin family. EMBO J 2002, 21:835-844.

20. Hubbert C, Guardiola A, Shao R, Kawaguchi Y, Ito A, Nixon A, Yoshida M, Wang XF, Yao TP: HDAC6 is a microtubule-associated deacetylase. Nature 2002, 417:455-458.

21. Mitsiades N, Mitsiades CS, Poulaki V, Chauhan D, Fanourakis G, Gu X Bailey C, Joseph M, Libermann TA, Treon SP, Munshi NC, Richardson PG, Hideshima T, Anderson KC: Molecular sequelae of proteasome inhibition 
in human multiple myeloma cells. Proc Natl Acad Sci U S A 2002,

99:14374-14379.

22. Sato A, Asano T, Ito K, Asano T: Vorinostat and bortezomib synergistically cause ubiquitinated protein accumulation in prostate cancer cells. J Urol 2012, 188:2410-2418.

23. Richardson PG, Schlossman RL, Alsina M, Weber DM, Coutre SE, Gasparetto C, Mukhopadhyay S, Ondovik MS, Khan M, Paley CS, Lonial S: PANORAMA 2: panobinostat in combination with bortezomib and dexamethasone in patients with relapsed and bortezomib-refractory myeloma. Blood 2013, 122:2331-2337.

doi:10.1186/1471-2490-14-71

Cite this article as: Sato et al:: Panobinostat synergizes with bortezomib to induce endoplasmic reticulum stress and ubiquitinated protein accumulation in renal cancer cells. BMC Urology 2014 14:71.

\section{Submit your next manuscript to BioMed Central and take full advantage of:}

- Convenient online submission

- Thorough peer review

- No space constraints or color figure charges

- Immediate publication on acceptance

- Inclusion in PubMed, CAS, Scopus and Google Scholar

- Research which is freely available for redistribution 